\title{
PARADOXICAL DECOMPOSITIONS AND INVARIANT MEASURES
}

\author{
PIOTR ZAKRZEWSKI
}

(Communicated by Andreas R. Blass)

\begin{abstract}
Suppose $G$ is a certain group of bijections of a given set $X$. A subset $E$ of $X$ is countably $G$-paradoxical if it contains disjoint subsets $A$, $B$ each of which can be taken apart into countably many pieces that may be rearranged via $G$ to form a partition of $E$. We prove that the existence of a countably additive measure on $P(X)$ that normalizes $X$ and vanishes on all countably $G$-paradoxical sets implies the existence of a countably additive, $G$-invariant measure on $P(X)$ normalizing $X$.
\end{abstract}

\section{TERMINOLOGY}

Our set-theoretic notation and terminology are standard. Ordinals are identified with sets of their predecessors and cardinals with initial ordinals. In particular, $\omega$ denotes the set of natural numbers and the first infinite cardinal. If $A$ is a set, then $P(A)$ denotes the family of all subsets of $A$ and $|A|$ the cardinality of $A$. If $f: X \rightarrow Y$ is a function and $A \subset X$, then $f[A]$ denotes the image of $A$ and $f \mid A$ the restriction of $f$ to $A$.

All measures considered in this paper are allowed to have values in $[0,+\infty]$. We are primarily concerned with countably additive measures defined on $P(X)$ and normalizing $X$. If $\mu$ is such a measure, then the family $I_{\mu}$ of all sets of $\mu$-measure 0 is called the ideal of $\mu$. It should be noted that $\mu$ may either be concentrated on (at most countably many) points of $X$ or, possibly, not. In the latter case, however, its existence requires the cardinality of $X$ to be at least real-valued measurable. Nevertheless, in both cases the ideal of $\mu$ is $\omega_{2}$-complete; i.e., every union of less than $\omega_{2}$ sets of measure 0 has measure 0 (see, e.g., $[4$, p. 300$]$ ).

Throughout the paper $G$ is always a certain group of bijections of a given set $X$. We express this equivalently by saying that $G$ acts on $X$.

Our terminology concerning paradoxical decompositions agrees with Wagon [6].

We say that sets $A, B \subset X$ are $G$-equidecomposable, $A \stackrel{G}{\sim} B$, (resp. countably $G$-equidecomposable, $A \underset{\omega}{G} B$ ), if $A$ and $B$ can each be partitioned into the same finite (resp. countable) number of respectively $G$-congruent pieces.

Received by the editors August 24, 1989.

1980 Mathematics Subject Classification (1985 Revision). Primary 03E05; Secondary 28C10.

Key words and phrases. Invariant measure, paradoxical set, real-valued measurable cardinal. 
We say that a set $E \subset X$ is $G$-paradoxical (resp. countably $G$-paradoxical) if $E$ contains disjoint sets $A, B$ such that $A \stackrel{G}{\sim} E$ and $B \stackrel{G}{\sim} E$ (resp. $A \underset{\omega}{\stackrel{G}{\sim}} E$ and $B \underset{\omega}{\stackrel{G}{\sim}} E$ ).

If $Z \subset X$, then we say that a measure on $P(Z)$ is $G$-invariant if it assigns the same measure to $G$-congruent subsets of $Z$.

Some special notation will be used to describe the action of $G$ on $X$. If $F \subset G$, then $[F]$ denotes the subgroup of $G$ generated by $F$. If $f \in G$, then the set $\{x \in X: f(x)=x\}$ of all fixed points of $f$ will be denoted by $\operatorname{fix}(f)$.

Suppose $H$ is a subgroup of $G$. If $x \in X$, then $H x$ denotes the set $\{h(x): h \in H\}$ called the $H$-orbit of $x$. For a cardinal $\kappa$ we set

$$
O_{\kappa}(H)=\{x \in X:|H x|=\kappa\}
$$

and

$$
S_{\kappa}(G)=\left\{O_{\kappa}(H): H=\text { a subgroup of } G \text { of cardinality } \kappa\right\} \text {. }
$$

\section{Preliminaries}

This paper is motivated by the following theorem of Tarski (see [5, p. 232]):

1.1. Theorem. Suppose $E$ is a subset of $X$. Then the following are equivalent:

(i) There is a finitely additive, G-invariant measure on $P(X)$ that normalizes $E$.

(ii) $E$ is not G-paradoxical.

It is easy to see that if $m$ is a finitely additive, $G$-invariant measure on $P(X)$ with $m(E)<+\infty$, and $E$ is $G$-paradoxical, then $m(E)=0$. The nontrivial part of Tarski's theorem asserts the existence of an invariant measure in the absence of a paradoxical decomposition.

The question arises whether there are any similar connections between the existence of a countably additive, $G$-invariant measure on $P(X)$ and the behavior of countably $G$-paradoxical subsets of $X$. Chuaqui [1] conjectured that Tarski's theorem is valid for this case also, but then he discovered a counterexample [2]. The point is that there may be no countably additive measure on $P(X)$ normalizing $X$ and vanishing on all countably $G$-paradoxical sets, for purely set-theoretic reasons which go far beyond the obvious obstacle that $X$ itself may be countably $G$-paradoxical.

Chuaqui [3] made another attempt to generalize Tarski's theorem, proving the following:

1.2. Theorem. Suppose $I$ consists of all $A \subset X$ such that $X$ contains pairwise disjoint subsets $\left\{A_{n}: n \in \omega\right\}$ with each $A_{n} \underset{\omega}{G} A$. Then the following are equivalent:

(i) There is a countably additive, G-invariant measure on $P(X)$ that normalizes $X$. 
(ii) There is a countably additive measure $\mu$ on $P(X)$ such that $\mu(X)=1$, $\mu$ vanishes on $I$, and the ideal of $\mu$ is $G$-invariant.

Chuaqui (quoted in Wagon [6, p. 136 and p. 232]) has conjectured that the above is valid without the assumption in (ii) that the ideal of $\mu$ is $G$-invariant.

In this paper we prove that Chuaqui's conjecture is true, obtaining a countably additive $G$-invariant measure on $P(X)$ of total measure 1 directly from the assumption that there is a countably additive measure on $P(X)$ that normalizes $X$ and vanishes on all countably $G$-paradoxical sets. This seems to be the closest possible counterpart of Tarski's theorem in the countably additive case.

From now on we assume that all measures under consideration are countably additive.

Our arguments rely on the following basic lemma (cf. [8, proof of Theorem 2.2]).

1.3. Lemma. Suppose $\mu$ is a measure on $P(X)$ normalizing $X$. If $S_{\omega_{1}}(G) \subset$ $I_{\mu}$, then every subset of $X$ of positive measure contains a set $A$ with an associated family $H \subset G$ satisfying the following conditions:

(1) $\mu(A)>0$ and $|H|<\omega_{1}$,

(2) for every $g \in G$,

$$
\mu\left(A \cap g^{-1}[A] \backslash \mathrm{fix}(g)\right)=0,
$$

and

$$
\mu\left(A \cap g^{-1}\left[X \backslash \bigcup_{h \in H} h[A]\right]\right)=0
$$

We shall need one more auxiliary fact (see [7, Lemma 1.1]).

1.4. Proposition. Let $E$ be a subset of $X$. Then every $G$-invariant measure on $P(E)$ that normalizes $E$ can be extended to a $G$-invariant measure on $P(X)$.

\section{Results}

The principal aim of this section is to prove the following:

2.1. Theorem. The following are equivalent:

(i) There exists a $G$-invariant measure on $P(X)$ that normalizes $X$.

(ii) There exists a measure on $P(X)$ that normalizes $X$ and vanishes on all countably $G$-paradoxical subsets of $X$.

We begin with a useful characterization of countably $G$-paradoxical subsets of $X$. 
2.2. Lemma. Suppose $E$ is a subset of $X$. Then the following are equivalent:

(i) $E$ is countably G-paradoxical.

(ii) There exists a countable subgroup $H$ of $G$ such that $|H x \cap E|=\omega$ for every $x \in E$.

Proof (i) $\Rightarrow$ (ii). Assume that $E$ is countably $G$-paradoxical. Then it is not hard to see that $E$ contains infinitely many pairwise disjoint subsets $\left\{A_{n}: n \in\right.$ $\omega\}$ with each $A_{n} \underset{\omega}{\stackrel{G}{\omega}} E$. For every $n \in \omega$, let $F_{n}$ be the countable family of functions from $G$ that have been used to obtain $A_{n}$ from pieces of $E$. Define $H=\left[\bigcup_{n \in \omega} F_{n}\right]$. Clearly, $H$ is as required.

(ii) $\Rightarrow$ (i). Assume $H$ is a countable subgroup of $G$ such that $|H x \cap E|=\omega$ for every $x \in E$. Let $\left\{S_{n}: n \in \omega\right\}$ be a partition of $E$ into selectors of the family $\{H x \cap E: x \in E\}$. It is easy to see that $S_{n} \underset{\omega}{\stackrel{H}{\sim}} S_{m}$ for each $n, m \in \omega$. It follows that, for any infinite subset $T$ of $\omega, \bigcup_{n \in T} \underset{n}{S_{\omega}} \underset{\omega}{H} E$. Consequently, $E$ is countably $G$-paradoxical.

Now the main problem that we face is how to define a $G$-invariant measure on $P(X)$ that normalizes $X$. The next definition is intended to describe a class of cases in which this is not hard to do.

2.3. Definition. Let $\mu$ be a measure on $P(X)$ normalizing $X$. We say that the action of $G$ on $X$ is $\mu$-essentially finite if there exists an at most countable subgroup $F$ of $G$ satisfying the following conditions:

(1) $\mu\left(O_{\omega}(F)\right)=0$,

(2) $\mu(\{x \in X: g(x) \notin F x\})=0$ for each $g \in G$.

We say that the action of $G$ is essentially finite if it is $\mu$-essentially finite for a certain measure $\mu$, as above.

2.4. Lemma. If the action of $G$ on $X$ is essentially finite, then there exists a $G$-invariant measure on $X$ that normalizes $X$.

Proof. Let $\mu$ and $F$ witness that the action of $G$ is essentially finite. By condition (1) of 2.3, $\mu\left(O_{n}(F)\right)>0$ for a certain $n \in \omega$.

Let $S$ be a selector of the family of all $F$-orbits of length $n$. Since $O_{n}(F)=$ $\bigcup_{f \in F} f[S]$, we can assume without loss of generality that $\mu(S)>0$. Define a measure $m$ on $P(X)$ by

$$
m(A)=\frac{1}{n \cdot \mu(S)} \cdot \sum_{k=1}^{n} k \cdot \mu(\{x \in S:|A \cap F x|=k\})
$$

for $A \subset X$.

Clearly, $m$ is $F$-invariant. To see that it is $G$-invariant, take an arbitrary $A \subset X$ and $g \in G$. Set $A_{1}=\{x \in A: g(x) \in F x\}$. It is not hard to see that $g\left[A_{1}\right] \underset{\omega}{F} A_{1}$. Consequently, $m\left(g\left[A_{1}\right]\right)=m\left(A_{1}\right)$. Now set $A_{2}=A \backslash A_{1}$. We 
have

$$
\left\{x \in S: A_{2} \cap F x \neq \varnothing\right\} \subset \bigcup_{f \in F}\{x \in X:(g \circ f)(x) \notin F x\} .
$$

So, by condition (2) of 2.3 ,

$$
\mu\left(\left\{x \in S: A_{2} \cap F x \neq \varnothing\right\}\right)=0 .
$$

It follows that $m\left(A_{2}\right)=0$. But $g\left[A_{2}\right] \subset\left\{x \in X: g^{-1}(x) \notin F x\right\}$, so a similar argument shows that $m\left(g\left[A_{2}\right]\right)=0$.

Consequently, $m(g[A])=m(A)$, as required.

The following lemma is the key point of our argument.

2.5. Lemma. Let $\mu$ be a measure on $P(X)$ normalizing $X$. Then the following are equivalent:

(i) The action of $G$ is $\mu$-essentially finite.

(ii) $\mu$ vanishes on all countably $G$-paradoxical subsets of $X$.

Proof (i) $\Rightarrow$ (ii). Let $F$ witness that the action of $G$ is $\mu$-essentially finite. Take an arbitrary countably $G$-paradoxical subset $E$ of $X$. By 2.2 , there is a countable subgroup $H$ of $G$ such that $|H x \cap E|=\omega$ for any $x \in E$. Then, since $O_{\omega}(H) \backslash O_{\omega}(F) \subset \bigcup_{h \in H}\{x \in X: h(x) \notin F x\}$, it follows from conditions (1) and (2) of 2.3. that $\mu\left(O_{\omega}(H)\right)=0$. But $E \subset O_{\omega}(H)$, so $\mu(E)=0$.

(ii) $\Rightarrow\left(\right.$ i) . By 2.2, $S_{\omega}(G)$ consists of countably $G$-paradoxical subsets of $X$. So, by the hypotheses, $S_{\omega}(G) \subset I_{\mu}$. It also follows that $S_{\omega_{1}}(G) \subset I_{\mu}$. To see this, take an arbitrary subgroup $H$ of $G,|H|=\omega_{1}$, and let $H=$ $\left\{h_{\xi}: \xi<\omega_{1}\right\}$. For each $\alpha, \alpha<\omega_{1}$, set $H_{\alpha}=\left[\left\{h_{\xi}: \xi<\alpha\right\}\right]$. Then $O_{\omega_{1}}(H) \subset \bigcup_{\omega \leq \alpha<\omega_{1}} O_{\omega}\left(H_{\alpha}\right)$, so, by $\omega_{2}$-completeness of $I_{\mu}$, we conclude that $O_{\omega_{1}}(H) \subset I_{\mu}$.

Now let $\mathscr{R}$ be a maximal subset of $P(X) \times P(G)$ satisfying the following conditions:

(1) If $\langle A, H\rangle \in \mathscr{R}$, then $\mu(A)>0$ and $|H|<\omega_{1}$.

(2) If $\langle A, H\rangle \in \mathscr{R}$, then for each $g \in G$ we have $\mu\left(A \cap g^{-1}[A] \backslash\right.$ fix $\left.(g)\right)=$ 0 , and $\mu\left(A \cap g^{-1}\left[X \backslash \bigcup_{h \in H} h[A]\right]\right)=0$.

(3) If $\left\langle A_{1}, H_{1}\right\rangle,\left\langle A_{2}, H_{2}\right\rangle \in \mathscr{R}$ and $\left\langle A_{1}, H_{1}\right\rangle \neq\left\langle A_{2}, H_{2}\right\rangle$, then $A_{1} \cap A_{2}=$ $\varnothing$.

By 1.3, $\mathscr{R} \neq \varnothing$. Also, by conditions (1) and (3), $|\mathscr{R}| \leq \omega$, so let $\mathscr{R}=$ $\left\{\left\langle A_{n}, H_{n}\right\rangle: n \in \omega\right\}$. Set $F=\left[\bigcup_{n \in \omega} H_{n}\right]$. We claim that $F$ witnesses that the action of $G$ is $\mu$-essentially finite. To see this, take an arbitrary $g \in G$. For each $n \in \omega$, let $H_{n}=\left\{h_{n, k}: k \in \omega\right\}$; then

$$
\begin{aligned}
\left\{x \in A_{n}:(\forall k \in \omega) g(x) \neq h_{n, k}(x)\right\} \\
\subset\left(A_{n} \cap g^{-1}\left[X \backslash \bigcup_{k \in \omega} h_{n, k}\left[A_{n}\right]\right]\right) \\
\cup \bigcup_{k \in \omega}\left(A_{n} \cap\left(g^{-1} \circ h_{n, k}\right)\left[A_{n}\right] \backslash \mathrm{fix}\left(g^{-1} \circ h_{n, k}\right)\right),
\end{aligned}
$$


so by condition (2), $\mu\left(\left\{x \in A_{n}:(\forall k \in \omega) g(x) \neq h_{n, k}(x)\right\}\right)=0$. But, by 1.3 and the maximality of $\mathscr{R}, \mu\left(X \backslash \cup_{n \in \omega} A_{n}\right)=0$. Consequently,

$$
\mu(\{x \in X: g(x) \notin F x\})=0 \text {, }
$$

as required.

Now we are ready to complete the proof of 2.1 . The implication (i) $\Rightarrow$ (ii) is obvious: any $G$-invariant measure on $P(X)$ normalizing $X$ vanishes on all countably $G$-paradoxical subsets of $X$. The reverse implication follows immediately from 2.4 and 2.5 .

It is not difficult to generalize 2.1 to the case in which the measure is required to be equal to 1 not on $X$ but on another set $E \subset X$.

2.6. Theorem. Let $E$ be a subset of $X$. Then the following are equivalent:

(i) There exists a G-invariant measure on $P(X)$ that normalizes $E$.

(ii) There exists a measure on $P(E)$ that normalizes $E$ and vanishes on all countably G-paradoxical subsets of $E$.

Proof. Again, only the implication (ii) $\Rightarrow$ (i) requires proof. It is based on the following:

2.7. Lemma. There exists a group $\widetilde{G}$ of bijections of $E$ satisfying the following conditions:

(1) Every countably $\widetilde{G}$-paradoxical subset of $E$ is countably G-paradoxical.

(2) Every $\widetilde{G}$-invariant measure on $P(E)$ is G-invariant.

Proof of 2.7. Let $\mathscr{R}$ be the family of all ordered pairs $\langle g, A\rangle$ such that $g \in G$, $A \subset E, g[A] \subset E$, and $A \cap g[A]=\varnothing$. For each $\langle g, A\rangle \in \mathscr{R}$, define the bijection $g^{A}$ of $E$ by

$$
g^{A}(x)= \begin{cases}g(x), & \text { if } x \in A, \\ g^{-1}(x), & \text { if } x \in g[A], \\ x, & \text { if } x \in E \backslash(A \cup g[A]) .\end{cases}
$$

Let $\widetilde{G}$ be the group of bijections of $E$ generated by the family $\left\{g^{A}:\langle g, A\rangle \in\right.$ $\mathscr{R}\}$.

To see that $\widetilde{G}$ satisfies condition (1), take an arbitrary countably $\widetilde{G}$-paradoxical subset $A$ of $E$. By 2.2 , there is a countable subgroup $\widetilde{H}$ of $\widetilde{G}$ such that $|\widetilde{H} x \cap A|=\omega$ for every $x \in A$. Now, notice that for each $g \in \widetilde{G}$ there exists a finite family $F(g) \subset G$ such that $\forall x \in E(g(x) \neq x \Rightarrow \exists f \in F(g) g(x)=f(x))$. Set $H=\left[\bigcup_{g \in \widetilde{H}} F(g)\right]$; then $|H| \leq \omega$ and $|H x \cap A|=\omega$ for each $x \in A$. So, by $2.2, A$ is countably $G$-paradoxical.

To see that $\widetilde{G}$ satisfies condition $(2)$, consider a $\widetilde{G}$-invariant measure $m$ on $P(E)$. Take an arbitrary $A \subset E$ and $g \in G$ such that $g[A] \subset E$. Set $H_{1}=[\{g\}]$ and let $\left\{S_{n}: n \in \lambda\right\}, \lambda \leq \omega$, be a partition of $X$ into (sub-)selectors of the family of all $H_{1}$-orbits. For each $n \in \lambda$, put $A_{n}=(A \backslash \mathrm{fix}(g)) \cap S_{n}$; since 
$A_{n} \cap g\left[A_{n}\right]=\varnothing$ and $m$ is $\widetilde{G}$-invariant, we have $m\left(g\left[A_{n}\right]\right)=m\left(g^{A_{n}}\left[A_{n}\right]\right)=$ $m\left(A_{n}\right)$. Consequently,

$$
\begin{aligned}
m(A \backslash \mathrm{fix}(g)) & =\sum_{n \in \lambda} m\left(A_{n}\right)=\sum_{n \in \lambda} m\left(g\left[A_{n}\right]\right) \\
& =m(g[A] \backslash \mathrm{fix}(g)),
\end{aligned}
$$

so finally $m(A)=m(g[A])$ and the proof of 2.7 is completed.

Now we are ready to finish the proof of 2.6. Let $\mu$ be a measure on $P(E)$ that normalizes $E$ and vanishes on all countably $G$-paradoxical subsets of $E$. By $2.7, \mu$ also vanishes on all countably $\widetilde{G}$-paradoxical subsets of $E$, so by 2.1 , there exists a $\widetilde{G}$-invariant measure $m$ on $P(E)$ with $m(E)=1$. Again by 2.7, $m$ is $G$-invariant, so by 1.4 , it can be extended to a $G$-invariant measure on $P(X)$.

2.8. Remark. The notion of countable equidecomposability may be generalized to the case in which pieces of decompositions are restricted to a given $G$-invariant $\sigma$-algebra $\mathscr{A}$ of subsets of $X$. The theorem of Chuaqui (1.2) is still valid in this setting after appropriate changes; i.e., measures are defined on $\mathscr{A}$, and $I$ consists of all $A \in \mathscr{A}$ such that $X$ contains countably many pairwise disjoint copies of $A$ in the sense of countable $G$-equidecomposability with pieces from $\mathscr{A}$. But the question of whether Chuaqui's conjecture concerning this general situation is true remains open.

Added in proof. The author has recently proved that the answer is yes.

\section{REFERENCES}

1. R. B. Chuaqui, Cardinal algebras and measures invariant under equivalence relation, Trans. Amer. Math. Soc. 142 (1969), 61-79.

2. The existence of an invariant countably additive measures and paradoxical decompositions, Notices Amer. Math. Soc. 20 (1973), A636-A637.

3. 313-329.

4. T. Jech, Set theory, Academic Press, New York, 1978.

5. A. Tarski, Cardinal algebras, Oxford Univ. Press, New York, 1949.

6. S. Wagon, The Banach-Tarski paradox, Cambridge Univ. Press, Cambridge, 1985.

7. P. Zakrzewski, On universal semiregular invariant measures, J. Symbolic Logic 53 (1988), 1170-1176.

8. _ The existence of universal invariant measures on large sets, Fund. Math. 133 (to appear).

Institute of Mathematics, University of Warsaw, PKiN IXP., 00-901 WaRsaw, Poland 